\title{
Comment on "Existence Theorem for Integral and Functional Integral Equations with Discontinuous Kernels"
}

\author{
Krzysztof A. Topolski \\ Institute of Mathematics, University of Gdaìsk, Wit Stwosz 57, 80-952 Gdaǹsk, Poland \\ Correspondence should be addressed to Krzysztof A. Topolski; matkt@mat.ug.edu.pl \\ Received 17 June 2015; Accepted 23 June 2015 \\ Academic Editor: Dumitru Motreanu
}

Copyright (C) 2015 Krzysztof A. Topolski. This is an open access article distributed under the Creative Commons Attribution License, which permits unrestricted use, distribution, and reproduction in any medium, provided the original work is properly cited.

We present a counterexample to the main result of the abovementioned paper showing that this result is false and cannot be improved in a simple way.

\section{Introduction}

In [1] author considers the nonlinear Volterra integral equation (VIE)

$$
x(t)=u(t)+\int_{0}^{t} f(t, \tau, x(\tau)) d \tau
$$

and the nonlinear functional Volterra integral equation (FVIE)

$$
x(t)=u(t)+\int_{0}^{t} f(t, \tau, x(\tau), x) d \tau .
$$

Theorem 2.1 of [1] states the following.

Theorem 1. Let $u:[0,1] \rightarrow \mathbb{R}$ and let $f:[0,1] \times[0,1] \times \mathbb{R} \rightarrow$ $\mathbb{R}$ be given. Suppose that (C1)-(C4) are fulfilled.

(C1) $u$ is continuous.

(C2) For each $(t, x) \in[0,1] \times \mathbb{R}$, the function $\tau \rightarrow f(t, \tau, x)$ is Lebesgue measurable. For all $(t, x) \in[0,1] \times \mathbb{R}$ and for almost all $\tau \in[0,1]$,

$$
|f(t, \tau, x)|<M(\tau),
$$

where $M:[0,1] \rightarrow[0, \infty]$ is a Lebesgue integrable function.

(C3) For each $(t, \tau, x) \in[0,1] \times[0,1] \times \mathbb{R}$

$$
\limsup _{y \uparrow x} f(t, \tau, y) \leq f(t, \tau, x)=\liminf _{y \downarrow x} f(t, \tau, y) .
$$

(C4) Let $F=\left\{y \in \mathbb{R}:|y| \leq\|u\|+\left|\int_{0}^{1} M(\tau) d \tau\right|\right\}$, where $\|u\|=\max \{|u(t)| ; t \in[0,1]\}$. For every $y \in F$ and all $n \in \mathbb{N}$ the functions

$$
t \longrightarrow \int_{0}^{t} \sup _{|x-y| \leq 1 / 3^{n}} f(t, \tau, x) d \tau
$$

are equicontinuous and tend to zero as $t \downarrow 0$.

Under the above assumptions VIE expressed by (1) has extremal solutions in the interval $[0,1]$.

In the following we present a counterexample showing that this result is false.

\section{Comment on the Assumption (C4)}

Define

$$
h_{y, n}(t)=\int_{0}^{t} \sup _{|x-y| \leq 1 / 3^{n}} f(t, \tau, x) d \tau,
$$

$$
y \in F, n \in \mathbb{N}, t \in[0,1] .
$$

(C4) states that $h_{y, n}(t)$ is equicontinuous in $[0,1]$ and tends to zero as $t \downarrow 0$. In fact the last seems to be superfluous since it follows from equicontinuity (or from (C2)).

Assume that $f$ does not depend on $\tau$; that is, we set $f(t, \tau, x)=f(t, x)$ (with a small violation of notation). Now 
(C2) gives $|f(t, x)| \leq M$ in $[0,1] \times \mathbb{R}$ for some $M \geq 0$. We also have

$$
\begin{aligned}
h_{y, n}(t)=t \sup _{|x-y| \leq 1 / 3^{n}} f(t, x)= & \sup _{|x-y| \leq 1 / 3^{n}} t f(t, x), \\
& y \in F, n \in \mathbb{N}, t \in[0,1] .
\end{aligned}
$$

Proposition 2. If $f$ is Lipschitz continuous in $t$, that is, if there exists $L \geq 0$ such that

$$
|f(t, x)-f(\bar{t}, x)| \leq L|t-\bar{t}|
$$

for all $(t, x),(\bar{t}, x) \in[0,1] \times \mathbb{R}$ then $(C 4)$ is satisfied.

Proof. For all $t, \bar{t} \in[0,1]$ and $y \in F, n \in \mathbb{N}$, we have

$$
\begin{aligned}
& \left|h_{y, n}(t)-h_{y, n}(\bar{t})\right| \\
& =\left|\sup _{|x-y| \leq 1 / 3^{n}} t f(t, x)-\sup _{|x-y| \leq 1 / 3^{n}} \bar{t} f(\bar{t}, x)\right| \\
& \leq \sup _{|x-y| \leq 1 / 3^{n}}|t f(t, x)-\bar{t} f(\bar{t}, x)| \\
& \leq \sup _{|x-y| \leq 1 / 3^{n}}|t f(t, x)-\bar{t} f(t, x)| \\
& \quad+\sup _{|x-y| \leq 1 / 3^{n}}|\bar{t} f(t, x)-\bar{t} f(\bar{t}, x)| \\
& \leq M|t-\bar{t}|+L|t-\bar{t}|=(M+L)|t-\bar{t}| .
\end{aligned}
$$

This gives equicontinuity of $h_{y, n}(t)$.

\section{The Counterexample}

Our example is a modification of this given in [2]. Consider VIE

$$
z(s)=-s \int_{-1}^{s} z(\tau)^{1 / 2} d \tau \quad s \in[-1,1]
$$

where $z^{1 / 2}=|z|^{1 / 2} \operatorname{sgn} z$ for any $z \in \mathbb{R}$ (see [2]).

Consider VIE

$$
x(t)=(2-4 t) \int_{0}^{t} x(\tau)^{1 / 2} d \tau \quad t \in[0,1] .
$$

Proposition 3. Set $s=2 t-1, t \in[0,1]$, and $z(s)=x(t)$, $s \in[-1,1]$. A function $z(s)$ is a solution of $(10)$ if and only if $x(t)$ is a solution of (11).

Proof. Suppose that $z(s)$ is a solution of (10). Set $s=2 t-1$, $t \in[0,1]$, in (10). We have

$$
x(t)=z(2 t-1)=(1-2 t) \int_{-1}^{2 t-1} z(\tau)^{1 / 2} d \tau .
$$

By making a substitution $\tau=2 r-1$ in the integral we get

$$
\begin{aligned}
x(t) & =(2-4 t) \int_{0}^{t} z(2 r-1)^{1 / 2} d r \\
& =(2-4 t) \int_{0}^{t} x(r)^{1 / 2} d r .
\end{aligned}
$$

Hence, $x(t)$ satisfies (11). Similarly setting $t=(s+1) / 2, s \in$ $[-1,1]$, in (11) we obtain that $z(s)$ satisfies $(10)$ if $x(t)$ satisfies (11).

Corollary 4. VIE (10) has a maximal (minimal) solution if and only if (11) has a maximal (minimal) solution.

\section{Consider VIE}

$$
x(t)=(2-4 t) \int_{0}^{t}[I(x(\tau))]^{1 / 2} d \tau \quad t \in[0,1]
$$

where $I(x)=(\operatorname{sgn} x) \min \{|x|, 4\}$ for $x \in \mathbb{R}$.

Proposition 5. VIE's (11) and (14) have the same (nonempty) sets of solutions.

Proof. The statement follows from the fact that every solution of (11) and (14) takes its values in the interval $[-4,4]$ where $I(x)=x$. Indeed, if $x$ satisfies (11) and $\|x\|=\max \{|x(t)| ; t \in$ $[0,1]\}$ then we have

$$
\begin{aligned}
|x(t)| & \leq|2-4 t| \int_{0}^{t}|x(\tau)|^{1 / 2} d \tau \leq 2 \int_{0}^{1}\|x\|^{1 / 2} d \tau \\
& \leq 2\|x\|^{1 / 2}, \quad t \in[0,1]
\end{aligned}
$$

which implies $\|x\| \leq 2\|x\|^{1 / 2}$ and $\|x\| \leq 4$. Since $|I(x)| \leq|x|$ a similar estimation holds for (14). Of course a zero function is a solution of both equations.

Set $f(t, \tau, x)=f(t, x)=(2-4 t)[I(x)]^{1 / 2}$. Of course, $f$ is Lipschitz continuous in $t$. It is not difficult to verify (see Proposition 2) the following.

Remark 6. VIE (14) satisfies all the assumptions of Theorem 1.

Proposition 7. VIE (14) has no extremal solution in $[-1,1]$.

Proof. In view of Corollary 4 and Proposition 5 we only need to show that (10) has no extremal solutions. This was in fact done in [2] where the proof is rather long and complicated. For the reader's convenience, we present an original and short explanation.

Suppose that $v$ is not a trivial solution of the problem

$$
\begin{aligned}
& v^{\prime}(s)=-s^{1 / 2} v(s)^{1 / 2}, \\
& v(-1)=0
\end{aligned}
$$

$$
\text { in }[-1,1] \text {. }
$$

Such solution exists since this problem, in view of the classical theory, has many solutions. Suppose that $z_{M}(s)$ is a maximal solution of (10). Since $0, s v(s),-s v(s)$ are all solutions of (10) we have $z_{M}(s) \geq \max \{0, s v(s),-s v(s)\}$; hence $z_{M}(s) \geq 0$ and it is not identically zero. This gives $z_{M}(s)=-s \int_{-1}^{s} z_{M}(\tau)^{1 / 2} d \tau<$ 0 for some $s \in(0,1]$. This leads to a contradiction. We finish the proof by observing that the negative of a minimal solution of (10) must be its maximal solution. 
Remark 8. Of course, we can improve Theorem 1 by assuming that $f$ is nondecreasing in $x$. In this case however, (C3) is not necessary and (C4) can be reduced to a simpler one and the result is well-known.

Remark 9. Theorem 3.1 [1] (FVIE (2)) and Theorem 4.1 [1] (system of Volterra integral equation) are false since they generalize Theorem 1 (Theorem 2.1 [1]).

\section{Conflict of Interests}

The author declares that there is no conflict of interests regarding the publication of this paper.

\section{References}

[1] E. R. Hassan, "Existence theorem for integral and functional integral equations with discontinuous kernels," Abstract and Applied Analysis, vol. 2012, Article ID 232314, 14 pages, 2012.

[2] H. E. Gollwitzer and R. A. Hager, "The nonexistence of maximum solutions of Volterra integral equations," Proceedings of the American Mathematical Society, vol. 26, pp. 301-304, 1970. 


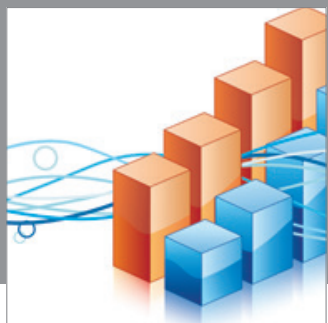

Advances in

Operations Research

mansans

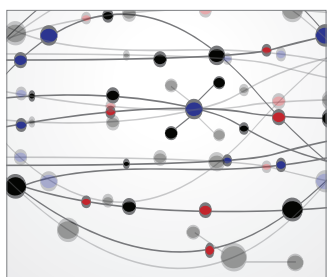

The Scientific World Journal
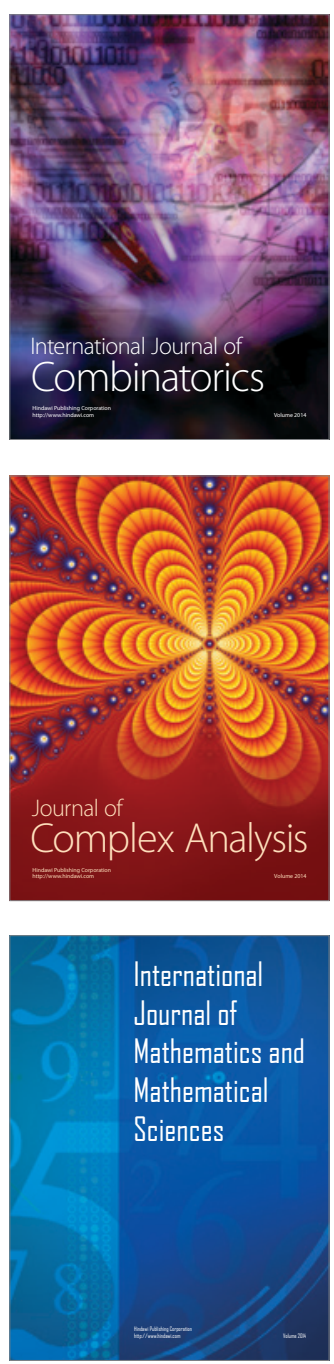
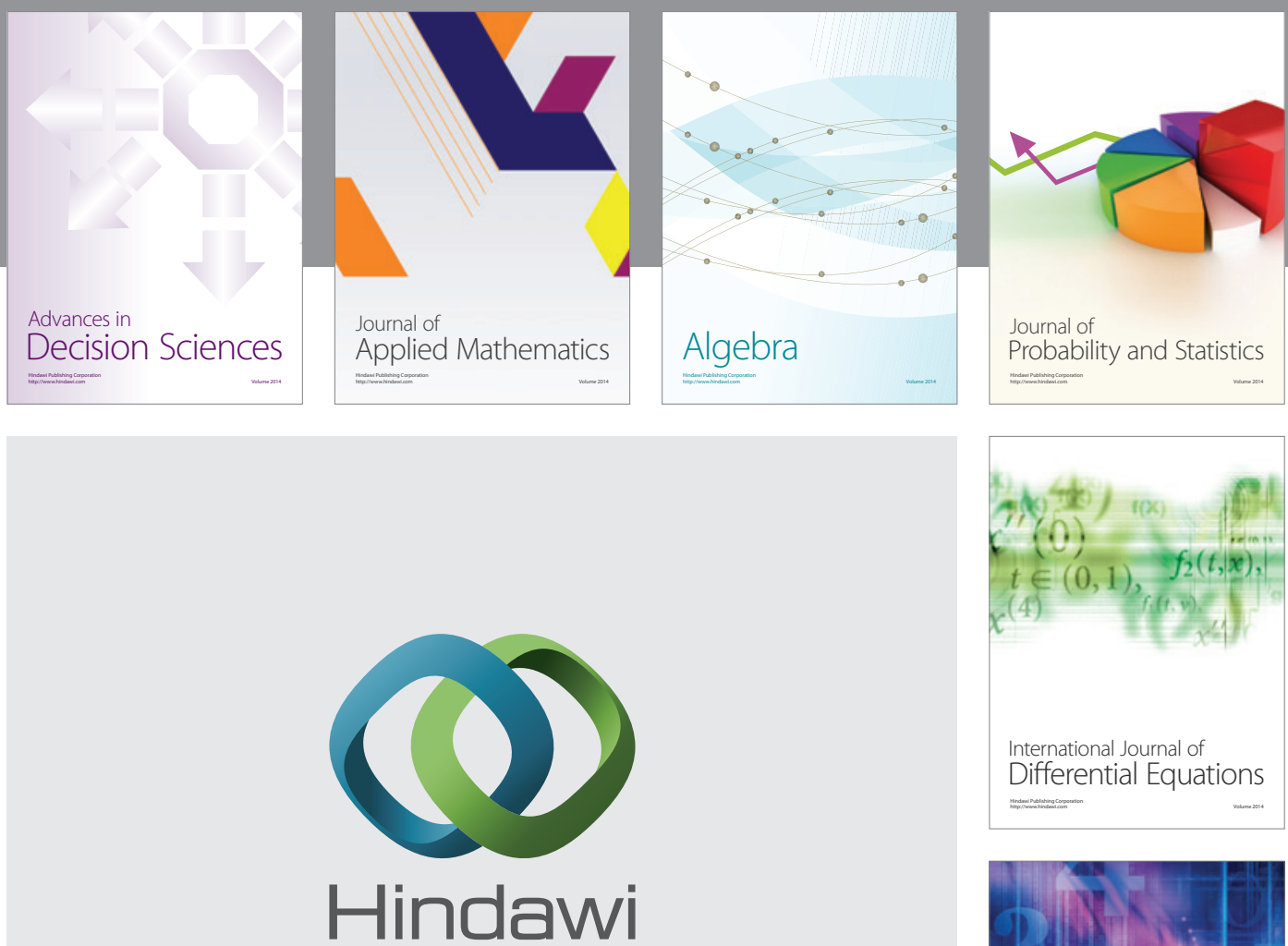

Submit your manuscripts at http://www.hindawi.com
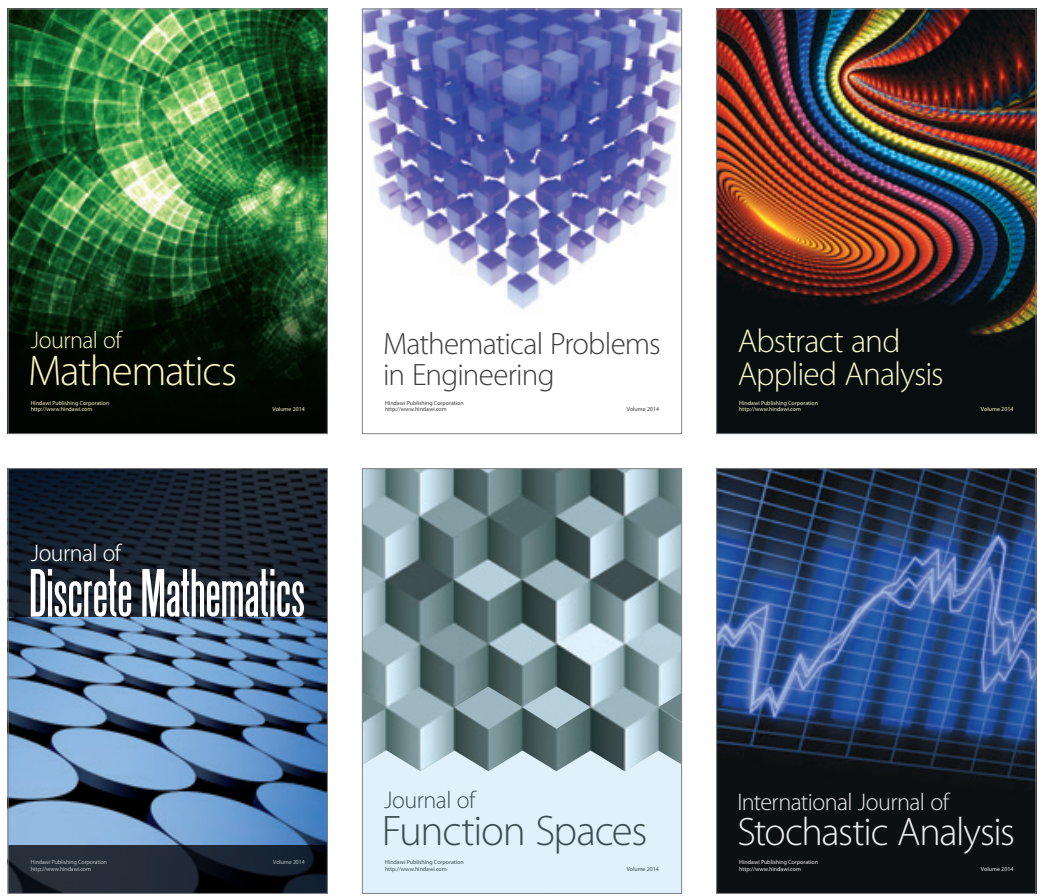

Journal of

Function Spaces

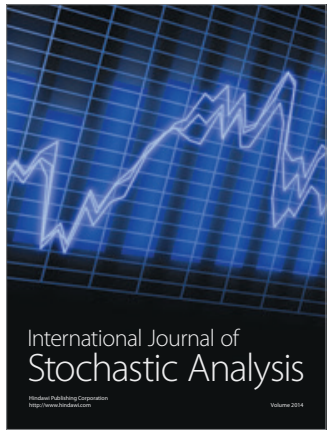

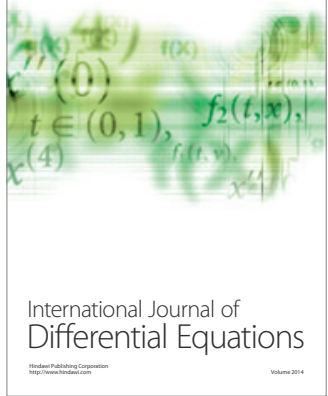
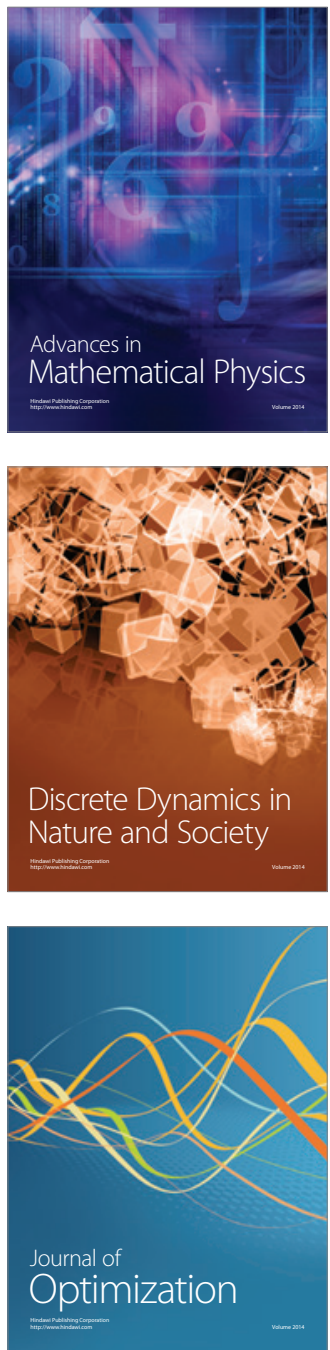